\title{
Transferencia del ácido linoleico conjugado (CLA) de la leche cruda a leche en polvo y condensada
}

\author{
Conjugated linoleic acid transfer \\ (CLA) from raw milk to powdered \\ milk and condensed milk
}

\begin{abstract}
The objective of this study was to assess the effect of processing fresh milk into dairy products on conjugated linoleic acid (CLA) content of total and individual CLA isomers (cis-9, trans-11; trans-10, cis-12; cis-10. cis-12) of dairy cow products, raw milk powdered and condensed milk. Additionally, samples of raw milk from two regions of southern Chile (Los Angeles and Los Lagos), obtained by a dairy plant were collected and analyzed. The processing of fresh milk dairy products affected the CLA and its isomers, finding a significant interaction between product type and sampling month, with the total average values of $1.35 \mathrm{~g} C L A$ $/ 100 \mathrm{~g}$ of fatty acids (AG) in fresh milk and 1.45 and $0.93 \mathrm{~g} / 100$ $g$ AG transferred to the milk powder and condensed respectively. As isomers, the cis-9, trans-11 had higher levels in the powdered milk that fresh milk in 7 months of the 10 months studied with values from 0.50 to $0.84 \mathrm{~g} / 100 \mathrm{~g}$ AG. The isomer trans- 10 , cis- 1 + cis-10, cis-12 CLA was found in a range of 0, 24 to $0.64 \mathrm{~g} / 100$ $g$ AG always low in fresh milk. It is concluded that further research is necessary to elucidate the precise effect of processing and storage of milk and milk products on the content of CLA needed. Key words: CLA; conjugated linoleic acid; milk; dairy products.
\end{abstract}

\section{INTRODUCCIÓN}

El ácido linoleico conjugado (CLA) se ha indicado como uno de los ácidos grasos (AG) más beneficiosos para la salud humana (1). De todos los isómeros el cis-9, trans -11 y trans-10, cis-12 han mostrado una actividad biológica interesante en salud humana (2). Los estudios en modelos animales y en líneas celulares han proporcionado evidencia de que el cis-9, trans-11, tiene un potente poder antiinflamatorio, es además inmunomodulador y actuaría como un producto anti obesidad y anticancerígeno, además de ser un biomarcador cardiovascular (3). Es por ello que los niveles de CLA en la leche y de los productos lácteos, son de especial interés nutricional por ser una fuente importante de estos AG (2).

El contenido de CLA en la grasa de alimentos derivados de especies rumiantes, depende de la actividad del ácido trans-vaccénico (TVA) y de la $\Delta 9$-desaturasa. Esta actividad a la vez está relacionada con el tipo de alimento que ingiere el animal, siendo mayor su producción cuando es alimentado a base de forraje fresco (4) y además, con dietas a base de oleaginosas, las cuales son ricas en ácido linoleico que aumenta la concentración de CLA en la grasa láctea (5). Sin embargo,
Juan Pablo Avilez R. Jorge Meyer R.

Escuela de Medicina Veterinaria, Facultad de Recursos Naturales. Universidad Católica de Temuco. Temuco. Chile

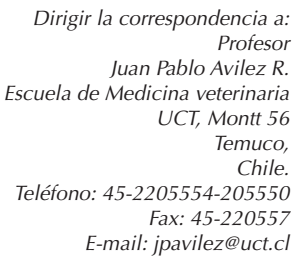

Este trabajo fue recibido el 31 de Marzo de 2014 y aceptado para ser publicado el 10 de Septiembre de 2014.

es contradictoria la cantidad de CLA encontrada en leche con la utilización de pradera más oleaginosa como ración base en rumiantes, reportándose aumentos en CLA con el uso de estos alimentos (6) o bien un nulo efecto al utilizar soya o canola como suplemento a la pradera $(7,8)$.

En el caso chileno se ha estudiado los AG en leche bovina recepcionada en planta, determinándose niveles de CLA de 1,750 g/100 g de AG en la Región de la Araucania y 1,410 g/100 g de AG para la Región del Bio-Bio; sin embargo, sólo se menciona al isómero cis- 9, trans-11 (9). Otro estudio donde se reportan los isómeros, cis-9, trans-11, trans-10, cis-12 y cis10, cis-12, señala niveles de CLA de 1,720 $\pm 1,207 \mathrm{~g} / 100 \mathrm{~g}$ de AG en silos de Osorno en época de primavera (10). Por otro lado, los productos lácteos presentaron niveles promedios muy variados en cuanto a sus contenidos de CLA, que van de 0,883 $\mathrm{g} / 100 \mathrm{~g}$ de $\mathrm{AG}$ en queso, 1,967 g/100 g AG en leche en polvo y a 1,502 g/100 g AG en mantequilla. En leche condensada se encontró niveles de 0,960 a 2,200 de CLA (10). A nivel internacional se ha reportado en quesos niveles de CLA que varían entre 0,400 a 1,700 g/100 g AG dependiendo si es queso gauda, jurassic u Old Emmentales (11). 
Estos productos lácteos usualmente sufren diversos cambios durante su preparación o procesamiento, el cual puede incluir tratamiento con calor intenso o moderado que puede llevar a cambios indeseables en lípidos o proteínas (12). Además se ha reportado que los lípidos de la leche pueden sufrir cambios químicos y físicos durante el procesamiento y almacenaje, tales como auto-oxidación y formación de AG trans (13). Sin embargo, no hay información en cuanto a la transferencia del CLA de leche fresca a producto.

Este estudio tuvo como objetivo determinar la cantidad de CLA y de sus isómeros, que se transfiere desde leche fresca a leche procesada, como es la leche condensada y la leche en polvo.

\section{MATERIAL Y MÉTODOS}

Las muestras experimentales

Las muestras de leche fueron recolectadas de dos regiones de Chile con dos sistemas productivos lecheros: uno basado en la alimentación a base de pradera fundamentalmente (Región de Los Lagos, Osorno) y el otro sistema basado en dietas TMR (ración total mezclada) (Región del Bío-Bío, Los Ángeles). Estas muestras de leche fresca se obtuvieron de los tanques de recepción de la empresa Nestlé-Chile, con una capacidad de $120.000 \mathrm{~L}$ y una temperatura de almacenamiento de $8{ }^{\circ} \mathrm{C}$. En Osorno había 6 tanques de recepción y en Los Ángeles 5 tanques.

Se recolectaron 3 muestras de leche cruda de los tanques de las plantas de Osorno y Los Ángeles a intervalos de 10 días, durante 10 meses $(n=90)$. Además, en forma paralela se tomaron 3 muestras de leche en polvo y leche condensada al mes $(n=30)$, producidas con la leche cruda muestreada. La leche en polvo se obtuvo por evaporación $\left(55-70{ }^{\circ} \mathrm{C}\right.$ ) y por atomización $\left(150-250^{\circ} \mathrm{C}\right.$ ) y la leche condensada por evaporación $\left(100^{\circ} \mathrm{C}\right)$. Estas muestras fueron recolectadas según el protocolo de la International Dairy Federation (14). Todas las muestras se enviaron en cajas de termo - aislado a $4{ }^{\circ} \mathrm{C}$ hasta el laboratorio de la UCTemuco y se almacenaron a $4{ }^{\circ} \mathrm{C}$ hasta su análisis.

\section{Contenido y la composición de CLA}

La determinación del CLA y sus isómeros (cis-9, trans11; trans-10, cis-12 y cis-10 cis-12) de la leche cruda, leche en polvo y leche condensada, se realizó primeramente extrayendo los lípidos totales por el método de Folch et al. (15), utilizando una mezcla de cloroformo y metanol (2:1, v: v). La metilación de los AG de las muestras fue hecho usando el método descrito por Morrison y Smith (16). Los esteres metílicos de los AG se analizaron mediante cromatografía de gases (HP 6890, Hewlett Packard, Surrey, Reino Unido), con detector de ionización de llama (FID) y columna capilar SP-2560 (100 m, $0.25 \mathrm{~mm}$ i.d. con 0,20 $\mu \mathrm{m}$ de espesor en la fase estacionaria; Supelco Inc., Bellefonte, Pensilvania, EE.UU.) con uso de gas He como trazador. La cromatografía de gases se usó en las siguientes condiciones: el volumen de inyección fue de 0,5 $\mu \mathrm{l}$, y se utilizó una inyección dividida (70:1, v: v); ultrapura de hidrógeno como gas portador. El inyector y las temperaturas del detector fueron de 250 y $300{ }^{\circ} \mathrm{C}$, respectivamente. La temperatura inicial fue de $70^{\circ} \mathrm{C}$ (durante $1 \mathrm{~min}$ ), aumentó en $5{ }^{\circ} \mathrm{C}$ por minuto a $100^{\circ} \mathrm{C}$ (durante $3 \mathrm{~min}$ ), aumentando en 10 ${ }^{\circ} \mathrm{C}$ por minuto hasta llegar a $175^{\circ} \mathrm{C}$ (durante $40 \mathrm{~min}$ ). Luego aumentó en $5{ }^{\circ} \mathrm{C}$ por minuto para llegar a $220{ }^{\circ} \mathrm{C}$ (durante 19 min) para un tiempo de ejecución total de 86,5 min. Los datos fueron cuantificados mediante el software Estacionario HPCHEM y expresado como un porcentaje de área de acuerdo con el total de AG identificados (7).

\section{Análisis estadístico}

El contenido de CLA y sus isómeros en leche y productos (leche en polvo y leche condensada) fueron analizados utilizando el modelo lineal general (GLM) del paquete estadístico SPSS 18.0 para Windows (SPSS Inc., Chicago, IL, EE.UU.): se determinó el efecto del tipo de producto (contenido de CLA en los productos lácteos) y el efecto del tipo de producto y mes (efecto en el contenido de CLA de procesar la leche cruda en productos lácteos). Las comparaciones por pares de medias se llevaron a cabo usando de pruebas de diferencia significativa de Tukey, considerando un nivel de significancia del 5\%.

\section{RESULTADOS}

En las cantidades de CLA total y cada uno de sus isómeros en la leche cruda recepcionada en las plantas lecheras de Osorno y Los Ángeles y las que se encuentran en los productos transformados a base a esta leche fresca, se observó que el CLA y su isómero cis-9, trans tiene una interacción significativa entre el tipo de producto y mes de muestreo (tabla 1). En general la leche cruda presentó un mayor contenido de CLA durante el otoño (marzo a mayo) y la primavera (octubre a

TABLA 1

Ácido linoleico conjugado (CLA) en leche fresca, leche en polvo y leche condensada.

$$
\text { CLA (g/100 g AG) }
$$

N

Total CLA

CLA cis-9, trans-11

CLA trans-10, cis-12

CLA cis-10, cis-12
Producto

Leche fresca

90
Leche en polvo

30
Efecto, $p^{1}$

Leche Condensada

$\mathrm{T}$

$M$

TxM

30

T. Tipo producto; M. Muestra mes; TxM. interacción; ${ }^{*} \mathrm{P}<0.05 ;{ }^{* *} \mathrm{P}<0.01$; ${ }^{* *} \mathrm{P}<0.001$; ns: no significativa, $\mathrm{P}>0.05$ 
diciembre) comparado con los meses de la época de invierno (junio a septiembre) que fueron más bajo. En contraste, la leche en polvo y la leche condensada, presentaron valores más altos durante los meses de invierno, aunque con una mayor variabilidad en estos productos, especialmente en la leche condensada (tabla 2).

Por otra parte, la leche condensada analizada presentó en la mayoría de los meses estudiados (7 de 10 meses de muestreo) un menor contenido de CLA que la leche cruda fresca; mientras que, en la leche en polvo el CLA, solo se mantuvo bajo en 4 de los 10 meses estudiados, encontrándose incluso en 6 meses aumentado o con valores similares de CLA en esta leche procesada en relación a la leche cruda. Los valores más altos de CLA en leche en polvo coinciden con los meses correspondientes a la estación de invierno.

En cuanto a los isómeros, se observa que el CLA cis-9, trans-11se presentó más alto en 7 meses de los 10 estudiados, en la leche en polvo en relación a la leche cruda fresca. En la leche condensada este isómero fue mayor en 5 meses. En cuanto al isómero CLA trans-10, cis-1+ CLA cis-10, cis-12 en 5 meses de los 10 estudiados, fue mayor en la leche en polvo con respecto a la leche fresca y en todas las muestras la leche condensada fue inferior a la leche fresca (tabla 2).

\section{DISCUSIÓN}

Se ha reportado valores de CLA en leche cruda recolectada en primavera de 1,72 g/100g AG en una planta lechera de Osorno y la cantidad promedio de CLA -en leche en polvo y de leche condensada- fue de 1,967 y 1,493 g/100g respectivamente (10). Estudios realizados en Europa han indicado que los valores máximos de CLA en la leche cruda es de 1,16 $\mathrm{g} / 100 \mathrm{~g}$ de AG para el caso de Alemania (17), 0,04 g/100 g de AG en Francia (18), y 0,72 g/100 g AG en Portugal (19).

Estos valores de CLA se asocian principalmente al tipo de alimentación que recibe el ganado lechero, siendo en los sistemas europeos basadas en dietas TMR y no en sistemas de pastoreo donde la alimentación base del ganado es el forraje (20). Los niveles de CLA en el caso de Chile también estarían asociados en la leche fresca y en los productos lácteos a la calidad y cantidad del forraje que consumen los animales, más que a dietas TMR o dietas ricas en oleaginosas $(7,8)$.

Se señala que la variación de la concentración de CLA en productos lácteos es esencialmente una función de su concentración original en la leche cruda; sin embargo, los resultados del efecto de las condiciones de procesamiento, almacenamiento y envasado en el contenido de CLA en diversos tipos de productos lácteos no son claros y prácticamente no existen trabajos del efecto del procesamiento en leche en polvo y en leche condensada. Se reporta, que los isómeros cis-9, trans-11 y cis-10, trans-12 no se verían afectados significativamente por el tratamiento de la leche en la elaboración de queso bajo proceso estándar (pasteurización de la leche y el posterior enfriamiento a $30^{\circ} \mathrm{C}$, con 6 meses de maduración a $8{ }^{\circ} \mathrm{C}$ ); sin embargo, este dato no es claro para la elaboración de leche en polvo y condensada, donde las temperaturas en el procesamiento es mayor que para el caso del queso (21).

Se ha observado una disminución notoria del ácido linoleico durante el almacenamiento, de $24,1 \%$ para leche UHT y $20,3 \%$ para leche en polvo, por lo que se concluye que los procesos aplicados, UHT y deshidratación, no resultaron en general en productos marcadamente diferentes entre sí (21). En el mismo sentido, se ha detectado una disminución de CLA total en muestras de queso después de 10 semanas de almacenamiento refrigerado a $4{ }^{\circ} \mathrm{C}$ (22). También existe una disminución de CLA de 21 a 53\% en los quesos calentados a $94 \pm 1,0{ }^{\circ} \mathrm{C}$ en un horno de microondas durante 5 a 10 minutos (12). Además, se ha observado una disminución de los isómeros trans de queso pasteurizada a $63^{\circ} \mathrm{C}$ durante 30 min o si éste se calienta en un microondas a $96{ }^{\circ} \mathrm{C}$ durante 5 min. (12). La disminución en el contenido de CLA en la leche después de calentamiento puede deberse a la acción de los

TABLA 2

Contenido de ácido linoleico conjugado (CLA, g/100 g AG ) en leche fresca y productos lácteos.

\begin{tabular}{|c|c|c|c|c|c|c|c|c|c|}
\hline \multirow[t]{2}{*}{ Mes } & \multicolumn{3}{|c|}{ CLA total } & \multicolumn{3}{|c|}{ CLA cis-9, trans-11 } & \multicolumn{3}{|c|}{ CLA trans -10 , cis $-12+C L A$ cis -10 , cis -12} \\
\hline & L. fresca & L. en polvo & L. cond. 1 & L. fresca & L. en polvo & L. cond. & L. fresca & L. en polvo & L. cond. \\
\hline Marzo & $1,53 \mathrm{~B}$ & $1,24 B$ & $1,61 \mathrm{~A}$ & $0,58 \mathrm{~b}, \mathrm{c}$ & $0,58 \mathrm{~b}, \mathrm{c}$ & $0,97 \mathrm{a}, \mathrm{A}$ & $0,95 a, A$ & $0,66 \mathrm{~b}, \mathrm{~B}$ & $0,64 b, A$ \\
\hline Abril & $1,33 \mathrm{~B}$ & $1,39 \mathrm{~B}$ & $1,05 \mathrm{~B}$ & $0,51 b, c$ & $0,81 \mathrm{a}, \mathrm{A}$ & $0,47 b, c$ & $0,83 a, B$ & $0,59 b, c$ & $0,58 \mathrm{~b}, \mathrm{~A}$ \\
\hline Mayo & $1,40 \mathrm{~B}$ & $1,39 \mathrm{~B}$ & $0,67 \mathrm{C}$ & $0,67 \mathrm{~b}, \mathrm{c}$ & $0,86 \mathrm{a}, \mathrm{A}$ & $0,30 c, C$ & $0,70 \mathrm{a}, \mathrm{B}$ & $0,53 b, c$ & $0,37 \mathrm{C}, \mathrm{B}$ \\
\hline Junio & $0,80 \mathrm{C}$ & $1,75 \mathrm{~A}$ & $0,80 \mathrm{C}$ & $0,24 b, D$ & $0,84 \mathrm{a}, \mathrm{A}$ & $0,43 \mathrm{~b}, \mathrm{c}$ & $0,56 b, c$ & $0,90 \mathrm{a}, \mathrm{A}$ & $0,37 \mathrm{C}, \mathrm{B}$ \\
\hline Julio & $1,46 \mathrm{~B}$ & $1,79 \mathrm{~A}$ & $1,00 \mathrm{~B}$ & $1,03 a, B$ & $0,89 a, A$ & $0,58 \mathrm{~b}, \mathrm{~B}$ & $0,44 b, c$ & $0,90 \mathrm{a}, \mathrm{A}$ & $0,43 b, B$ \\
\hline Agosto & $0,78 \mathrm{C}$ & $1,63 \mathrm{~A}$ & $1,06 \mathrm{~B}$ & $0,18 c, D$ & $0,78 a, A$ & $0,53 \mathrm{~b}, \mathrm{~B}$ & $0,64 b, c$ & $0,85 a, A$ & $0,53 \mathrm{~b}, \mathrm{~B}$ \\
\hline Septiembre & $0,99 \mathrm{~B}$ & $1,13 \mathrm{C}$ & $0,96 \mathrm{~B}$ & $0,25 b, D$ & $0,50 \mathrm{a}, \mathrm{C}$ & $0,50 \mathrm{a}, \mathrm{C}$ & $0,74 \mathrm{a}, \mathrm{C}$ & $0,63 \mathrm{a}, \mathrm{B}$ & $0,46 b, B$ \\
\hline Octubre & $2,15 \mathrm{~A}$ & $1,54 \mathrm{~A}$ & $0,54 \mathrm{C}$ & $1,32 a, A$ & $0,69 b, B$ & $0,29 c, D$ & $0,82 \mathrm{a}, \mathrm{C}$ & $0,85 a, A$ & $0,24 b, c$ \\
\hline Noviembre & $1,57 \mathrm{~B}$ & $1,34 \mathrm{~B}$ & $0,87 \mathrm{~B}$ & $0,41 \mathrm{~b}, \mathrm{c}$ & $0,63 \mathrm{a}, \mathrm{B}$ & $0,52 \mathrm{a}, \mathrm{B}$ & $1,16 a, A$ & $0,71 b, B$ & $0,35 c, C$ \\
\hline Diciembre & $1,03 \mathrm{C}$ & $1,42 \mathrm{~B}$ & $0,55 \mathrm{C}$ & $0,40 \mathrm{~b}, \mathrm{c}$ & $0,71 \mathrm{a}, \mathrm{B}$ & $0,19 c, D$ & $0,63 b, c$ & $0,72 \mathrm{a}, \mathrm{B}$ & $0,43 c, C$ \\
\hline
\end{tabular}

Medias dentro de filas $(a, b, c)$ o columna (A, B, C, D), con diferentes letras indican diferencias significativas $(p<0,05)$.

${ }^{1}$ Cond.: leche condensada. 
radicales libres que se forman como resultado de la oxidación de los lípidos (23).

Otros estudios han detectado nuevos isómeros de CLA en quesos madurados, los cuales se deberían a la biohidrogenación de ácido linolénico en este producto, lo que podría conducir a la formación de isómeros de CLA como productos intermedios $(24,25)$.

Por otra parte, el almacenamiento refrigerado y el tratamiento térmico resultaron en disminuciones significativas o desaparición de algunos de los isómeros de CLA que se encuentran en poca cantidad (isómeros menores de CLA), incrementándose en forma creciente los AG con isomería trans-trans, como también el cis-trans, trans-cis, y cis-cis especialmente en la leche en polvo enriquecida con CLA. Esto también se observó, en mezcla de leche fermentada, en yogur y en leche con sabor (26).

En el presente estudio se concluye, que el contenido de CLA aumentó en la leche en polvo, especialmente en los meses en los que la leche cruda original tenía valores CLA inferiores, lo que podría explicarse por el aumento cada vez mayor de los isómeros trans, aunque sólo se analizaron tres isómeros. Sin embargo, se necesitan más investigaciones para dilucidar el efecto exacto de las condiciones de procesamiento y almacenamiento de la leche y los productos lácteos en el contenido de CLA.

\section{RESUMEN}

El objetivo de este estudio fue evaluar la transferencia del ácido linoleico conjugado (CLA) y de sus isómeros (cis-9, trans-1; trans-10, cis-12; cis-10 cis-12) de leche cruda a productos lácteos como la leche en polvo y la leche condensada. Se recogieron y analizaron muestras de leche cruda de dos regiones del sur de Chile (Los Ángeles y Los Lagos), recepcionada por una planta de lácteos. El procesamiento de la leche fresca a productos lácteos afectó al CLA y a sus isómeros, encontrándose una interacción significativa entre el tipo de producto y el mes de muestreo, con valores promedios de CLA total de 1,35 g/100 g AG en la leche fresca y de 1,45 y 0,93 g/100 g AG traspasados a la leche en polvo y condensada respectivamente. En cuanto a los isómeros, el cis-9, trans-11 presentó niveles más altos en leche la en polvo que el leche fresca en 7 meses de los 10 estudiados con valores que fueron de 0,50 a 0,84 g/100 g AG. El CLA trans-10, cis-1+ CLA cis-10, cis-12 se encontró en un rango de 0, 24 a 0,64 a g/100 g AG siempre bajo de la leche fresca en el caso de la leche. Se concluye que es necesario una mayor investigación para dilucidar el efecto exacto del procesamiento y almacenamiento de la leche y los productos lácteos sobre el contenido de CLA.

Key words: CLA; ácido linoleico conjugado, leche, productos lácteos.

\section{BIBLIOGRAFÍA}

1. Pariza M. Park M. The biologically active isomers of conjugated linoleic acid. Prog Lipid Res. 2001; 40: 283-98.

2. Wahle K. Heys S. Rotondo D. Conjugated linoleic acids: are they beneficial or detrimental to health?. Prog Lipid Res. 2004; 43: 553-87.

3. Shingfield K. Chilliard Y, Toivonen V, Kairenius P, Givens D. Trans fatty acids and bioactive lipids in ruminant milk. In: Bioactive components of milk, Vol. 606. Springer-Verlag Berlin, Germany p. 3-65, 2008.

4. Butler G. Nielsen J. Slots T. Seal C. Eyre M. Sanderson R. Leifert $C$. Fatty acid and fat-soluble antioxidant concentrations in milk from high- and low-input conventional and organic systems: seasonal variation. J Sci Food Agr. 2008; 88: 1431-41.

5. Stanton C. Lawless F. Kjellmer G. Harrington D. Devery R. Connollyn F. Murphy J. Dietary influences on bovine milk cis-9, trans-11-conjugated linoleic acid content. J Food Sci. 1997; 62: 1083-86.

6. Lerch S. Ferlay A. Pomiès D. Martin B. Pires J. Chilliard $Y$. Rapeseed or linseed supplements in grass-based diets: effects on dairy performance of Holstein cows over two consecutive lactations. J Dairy Sci. 2012; 95: 1956-70.

7. Avilez J. Escobar P. Díaz C. Von fabeck G. Matamoros R. García F. Alonzo M. Delgado-Pertíñez M. Effect of extruded whole soybean dietary concentrate on conjugated linoleic acid concentration in milk in Jersey cows under pasture conditions. Span J Agric Res. 2012; 10: 409-18.

8. Avilez J. von Fabeck. G. García-Gómez F. Alonzo M. Delgado-Pertíñez M. Conjugated linoleic acid content in milk of Chilean Black Friesian cows under pasture conditions and supplemented with canola seed (Brassica napus) concentrate. Span J Agric Res. 2013; 11(3): 747-58.

9. Pinto M. Rubilar C. Carrasco E. Efecto estacional y del área geográfica en la composición de ácidos grasos en la leche de bovinos. Agro Sur 2002; 30 (2):75-90.

10. Avilez J. Vilches C. Alonzo M. Determinación de los niveles de ácido linoleico conjugado en alimentos lácteos en Chile. Rev Chil Nutr 2010; 36, N².

11. Bauman D. Griinari J. Regulation and nutritional manipulation of milk fat: Low-fat milk syndrome. Livest Prod Sci. 2001; 70: 15-29.

12. Herzallah S. Humeid M. Al-Ismail K. Effect of Heating and Processing Methods of Milk and Dairy Products on Conjugated Linoleic Acid and Trans Fatty Acid Isomer Content Dairy Sci. 2005; 88:1301-10.

13. Semma M. Trans fatty acids: Properties, benefits and risks. J. Health Sci. 2002; 48: 7-13.

14. International Dairy Federation. Milk and milk productsGuidance on sampling. FIL-IDF Standard 50 C. Int. Dairy Fed., Brussels, Belgium. 1995.

15. Folch J. Less H. Sloane-Stanley G. A simple method for the insolation and purification of total lipids from animal tissue. J Biol Chem. 1957; 726: 497-509.

16. Morrison W. Smith L. Preparation of fatty acids methyl esters and dimethylacetals from lipids with boron fluoridemethanol. J Lip Res. 1964; 5: 600-8.

17. Fritsche J. Steinhart $H$. Amounts of conjugated linoleic acid (CLA) in German foods and evaluation of daily intake. $Z$ Lebensm Unters Forsch A.1998; 206, 77-82.

18. Laloux L. du Chaffaut L. Razanamahefa L. Lafay L. Trans fatty acid content of foods and intake levels in France. Eur. J. Lipid Sci. Technol 2007; 109: 918-29.

19. Martins S. Lopes P. Alfaia C. Ribeiro V. Guerreiro T. Fontes C. Castro M. Soveral G. Prates J. Contents of conjugated linoleic acid isomers in ruminant-derived foods and estimation of their contribution to daily intake in Portugal. British J Nutr. 2007; 98: 1206-13.

20. Gómez-Cortés P. Frutos P. Mantecón A. Juárez M. De la Fuente $M$. Hervás G. Effect of supplementation of grazing dairy ewes with a cereal concentrate on animal performance and milk fatty acid profile. J Dairy Sci. 2009; 92: 3964-72.

21. Guzmán C. de Pablo E. Saturnino V. Yánez G. Carmen G. Zacarías H. Nieto S. Estudio comparativo de calidad de leche fluida y en polvo. Rev Chil Pediatr. 2003; 74 (3): 277-86.

22. Shantha N. Ram L. O'Leary J. Hicks C. Decker E. Conjugated linoleic acid concentrations in dairy products as affected 
by processing and storage. J Food Sci. 1995; 60: 695-7.

23. Leung Y. Liu. R. Trans-10, cis-12-conjugated linoleic acid isomer exhibits stronger oxyradical scavenging capacity than cis-9, trans-11-conjugated linoleic acid isomer. J Agricult Food Chem. 2000; 48: 5469-75.

24. Lavillonière F. Martin J. Bougnoux P. Sébédio J. Analysis of conjugated linoleic acid isomers and content in French cheeses. J Am Oil Chem Soc. 1998; 75: 343-52.

25. Sehat N. Kramer J. Mossoba M. Yurawecz M. Roach, J.
Eulitz K. Morehouse K. Кu Y. Identification of conjugated linoleic acid isomers in cheese by gas chromatography, silver ion high performance liquid chromatography and mass spectral reconstructed ion profiles. Comparison of chromatographic elution sequences. Lipids 1998; 33 : 963-71.

26. Rodríguez-Alcala L. Fontechal J. Hot Topic: Fatty Acid and Conjugated Linoleic Acid (CLA) Isomer Composition of Commercial CLA-Fortified Dairy Products: Evaluation After Processing and Storage. J Dairy Sci. 2007; 90: (5) 2083-90. 